Präv Gesundheitsf $2023 \cdot 18: 10-21$

https://doi.org/10.1007/s11553-021-00912-2

Eingegangen: 10. Mai 2021

Angenommen: 3. Oktober 2021

Online publiziert: 8. November 2021

(c) Der/die Autor(en) 2021

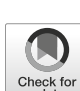

Saskia Redlof $(\mathbb{D}$ - Madlen Götz · Marlen Niederberger

Pädagogische Hochschule Schwäbisch Gmünd, Forschungsmethoden in der Gesundheitsförderung und Prävention, Schwäbisch Gmünd, Deutschland

\title{
Photovoice-Studie CORONA
}

\section{Erfahrungen aus dem Leben der Hochrisikogruppe während der Pandemie}

\begin{abstract}
Die Gefahr eines schwerwiegenden Krankheitsverlaufs bei einer Infektion mit COVID-19 (",coronavirus disease 2019“) ist für bestimmte Bevölkerungsgruppen deutlich erhöht. Dies führt dazu, dass den Betroffenen zu verstärkter Vorsicht im Alltag geraten wird und deutschlandweit verschiedene Maßnahmen initiiert werden, um gerade diese Gruppen zu schützen. Welche Auswirkungen Corona auf den Alltag der Hochrisikogruppe während der Pandemie hat, welche Ängste sie begleiten und woraus sie dennoch Kraft schöpfen können, wird in diesem Beitrag aus Sicht der Betroffenen diskutiert.
\end{abstract}

Personen mit bestimmten Grunderkrankungen sind einem deutlich erhöhten Risiko ausgesetzt, bei einer Infektion mit COVID-19 einen erschwerten Krankheitsverlauf zu entwickeln [8]. Zur Hochrisikogruppe zählen nach dem Robert Koch-Institut (RKI) u. a. Personen mit Erkrankungen des Herz-Kreislauf- und Atmungssystems, Diabetes, Krebserkrankungen etc. [20]. Aber auch weitere Faktoren, die den Gesundheitszustand beeinträchtigen, wie Adipositas, Rauchen, ein geschwächtes Immunsystem oder Personen im höheren Lebensalter (50 Jahre und älter) stuft das RKI als deutlich gefährdet ein. Schätzungen zur Folge ist rund ein Drittel der deutschen Bevölkerung ab 15 Jahren der Hochrisikogruppe zuzuordnen [22]. Dies sind häufig Personen mit niedrigem sozioökonomischem Status [27].

Um die Verbreitung von COVID-19 in der Bevölkerung einzudämmen, werden während der Coronapandemie durch die Politik, beratend und unterstützt von der Wissenschaft, kontinuierlich situationsangepasste Regelungen durchgesetzt, wie die Maskenpflicht oder die Schließung von Bildungseinrichtungen [2].

Durch die damit einhergehenden Veränderungen in der Alltagsgestaltung ist auch die Gesundheit derer betroffen, die nicht an COVID-19 erkranken [1]. So zeigt beispielsweise eine bundesweite Studie, dass die Coronakrise stark belastende Auswirkungen auf die psychische Gesundheit von Kindern und Jugendlichen hat [25]. Weitere Studien belegen negative Auswirkungen auf die Gesundheit und die Lebenszufriedenheit berufstätiger Eltern [10], Studierender bzw. Hochschulbeschäftigter sowie auf Erwerbstätige [17].

Die aktuell verfügbare Datenlage deutet an, dass sich bisherige Studien über Hochrisikopatient:innen auf Personen mit spezifischen Krankheitsbildern wie Herz- oder Krebserkrankungen konzentrieren $[13,16]$. Wie sich die Pandemie auf das Wohlbefinden von Hochrisikopatient:innen mit unterschiedlichen Grunderkrankungen auswirkt, widmet sich die folgende Studie.

\section{Das Forschungsprojekt}

Partizipative Gesundheitsforschung (PGF) gewinnt in Deutschland an Bedeutung [32]. Assoziierte Ziele sind positive Veränderungen auf die Gesundheit der Vertreter:innen einer Community (CoForschende) und die Unterstützung bei der Schaffung gesundheitsfördernder Verhältnissen in ihrer Lebenswelt [7].

Dem Ansatz der PGF folgen diverse Studien im Kontext von Corona, bei- spielsweise zum Thema Wohlbefinden von Studierenden oder Auswirkungen von Distanzlernen auf Kinder [11, 12]. Derartige Studien belegen auf einer methodischen Ebene, dass PGF auch unter Pandemiebedingungen (beispielsweise als Online-Format) durchführbar ist. Dies zeigt sich insbesondere bei Photovoice-Studien [3, 24]. Bei dieser Art der visuellen Datenerhebungsmethode erstellen Co-Forschende Fotos und ergänzen sie mit Geschichten aus ihrer Lebenswelt [28]. Damit assoziiert ist das Ziel, positive Veränderungen des Gesundheitszustandes der Community zu unterstützen [7].

Im vorliegenden Forschungsprojekt wurde Photovoice zur Analyse der Lebenswelt von Personen der Hochrisikogruppe während der Coronapandemie eingesetzt. Das Forscherteam bestand aus 3 Wissenschaftlerinnen, wobei eine Person selbst als Hochrisikopatientin gilt. Durch sie konnte ein erster niederschwelliger Kontakt zu möglichen Co-Forschenden hergestellt werden. Insbesondere ihre persönlichen Kontakte und der entfernte Bekanntenkreis der angesprochenen Personen wurden genutzt, um über die geplante Studie $\mathrm{zu}$ informieren und mögliche Co-Forschende zu gewinnen. Dadurch konnte das Forscherteam ohne einen öffentlichen Aufruf Hochrisikopatient:innen zur Studienteilnahme gewinnen. Die erste Kontaktaufnahme erfolgte über Telefon, E-Mail sowie durch persönliche Ansprache. Bei der Rekrutierung wurde auf ein möglichst breites Spektrum verschiedenster Grunderkrankungen und Lebensumstände geachtet (• Tab. 1), um eine gewisse Heterogenität der Stichpro- 


\begin{tabular}{|c|c|c|c|c|}
\hline Name & $\begin{array}{l}\text { Alter } \\
\text { (Jahre) }\end{array}$ & Geschlecht & Erkrankung & Lebenssituation \\
\hline \multirow[t]{3}{*}{ Magdalena } & \multirow[t]{3}{*}{25} & \multirow[t]{3}{*}{ W } & Diabetes Typ III & Alleinstehend \\
\hline & & & Neuroendokriner Tumor & Mehrpersonenhaushalt \\
\hline & & & Hashimoto & Studentin \\
\hline \multirow[t]{3}{*}{ Leo } & \multirow[t]{3}{*}{34} & \multirow[t]{3}{*}{ M } & Herzinsuffizienz & Alleinstehend \\
\hline & & & Chronische Niereninsuffizienz & Mehrpersonenhaushalt \\
\hline & & & bei Einzelniere & Technischer Berater \\
\hline \multirow[t]{3}{*}{ Regina } & \multirow[t]{3}{*}{45} & \multirow[t]{3}{*}{ W } & $\begin{array}{l}\text { PSC (primärsklerusierende } \\
\text { Cholangitis) }\end{array}$ & Verheiratet \\
\hline & & & Zystenniere & Zwei-Personen-Haushalt \\
\hline & & & Nur eine Niere veranlagt & Dozentin (Home-Office) \\
\hline \multirow[t]{2}{*}{ Theo } & \multirow[t]{2}{*}{58} & \multirow[t]{2}{*}{ M } & Metabolisches Syndrom & Verheiratet \\
\hline & & & $\begin{array}{l}\text { Folgeerkrankungen nach } \\
\text { Schlaganfall, Aneurysma }\end{array}$ & $\begin{array}{l}\text { Zwei-Personen-Haushalt } \\
\text { Frührentner }\end{array}$ \\
\hline \multirow[t]{3}{*}{ Margit } & \multirow[t]{3}{*}{62} & \multirow[t]{3}{*}{ W } & \multirow[t]{3}{*}{ Brustkrebs } & Verheiratet \\
\hline & & & & Zwei-Personen-Haushalt \\
\hline & & & & Lehrerin \\
\hline \multirow[t]{3}{*}{ Franziska } & \multirow[t]{3}{*}{79} & \multirow[t]{3}{*}{ W } & Herzschwäche & Verheiratet \\
\hline & & & Asthma & Zwei-Personen-Haushalt \\
\hline & & & Altersbedingte Krankheiten & Rentnerin \\
\hline \multirow[t]{3}{*}{ Josef } & \multirow[t]{3}{*}{83} & \multirow[t]{3}{*}{ M } & \multirow[t]{3}{*}{ Lungenödem } & Verheiratet \\
\hline & & & & Zwei-Personen-Haushalt \\
\hline & & & & Rentner \\
\hline
\end{tabular}

be zu gewährleisten. Dadurch konnten vielfältige Sichtweisen auf den Untersuchungsgegenstand exploriert werden. Einschlusskriterien waren hierbei, dass sich die Personen als Hochrisikopatient:innen wahrnehmen, ihren Alltag autonom gestalten und in keiner speziellen Pflegeeinrichtung leben.

\section{Projektdurchführung}

Nach der ersten Kontaktaufnahme mit den Co-Forschenden und dem Einholen einer informierten Zustimmung wurde das Projekt mit drei Online-Workshops durchgeführt, von denen zwei digital aufgezeichnet und transkribiert wurden (-Abb. 1). Das Forscherteam hat im Vorfeld der Studie an einer Moderationsschulung teilgenommen. Die Moderation erfolgte durch zwei der Wissenschaftlerinnen auf Basis eines Moderationsleitfadens (s. zusätzliches OnlineMaterial, abrufbar über ...) und unter Anwendung verschiedenster Reflexionsmethoden, wie etwa Blitzlicht oder Stimmungsbarometer. Die Darstellung wichti- wurde schriftlich protokolliert, um die Rückmeldungen mit in die Auswertung einfließen lassen zu können.

Bei den Gesprächen bilateral oder in der Gruppe wurden die Co-Forschenden immer wieder auf ihre Entscheidungsmacht im Projekt hingewiesen und deutlich gemacht, dass die Umsetzung und Ergebnisaufbereitung in ihren Händen liege. Das Forscherteam sei dazu da, zu unterstützen und mögliche Wege aufzuzeigen. Während der gesamten Studie wurde also darauf geachtet, dass die Co-Forschenden, den Partizipationsstufen von Wright [31] folgend, mindestens Mitbestimmung, möglichst aber Entscheidungsmacht erhielten. So haben die CoForschenden die Forschungsfragen selbst formuliert:

1. Wofür bin ich dankbar?

2. Was bedeutet Corona für mich?

Die Co-Forschenden nahmen Fotos zu den partizipativ herausgearbeiteten Fragestellungen auf und reflektieren diese innerhalb des strukturierten und moderierten Prozesses während der Workshops. Die Daten wurden über die Fotos, Geschichten, die Protokolle der bilateralen Gespräche und über die gemeinsamen Gruppendiskussionen (festgehalten über Transkriptionen) generiert. Ziel war es auch, die Co-Forschenden zu befähigen, ein tieferes Verständnis über die eigenen Bedarfe und Ressourcen, unter Pandemiebedingungen, zu erlangen [7].

\section{Auswertung}

Die wissenschaftliche Auswertung der Daten erfolgte durch die Wissenschaftlerinnen, unter Anwendung der zusammenfassenden Qualitativen Inhaltsanalyse [15]. Diese erfolgte zunächst unabhängig und wurde im Anschluss innerhalb des Teams der Wissenschaftlerinnen kritisch reflektiert und überarbeitet. Die Kategorien entsprechen den Geschichten der Co-Forschenden, die sie bei den Workshops gemeinsam entwickelt haben. Dazu gehören die von ihnen ausgewählten Fotos und Titel.

Innerhalb gemeinsamer Sitzungen der Wissenschaftlerinnen wurden die Aussagen der Co-Forschenden und mögliche Interpretationen kategorienbezogen dis- 
Präv Gesundheitsf 2023 · 18:10-21 https://doi.org/10.1007/s11553-021-00912-2

(c) Der/die Autor(en) 2021

\section{S. Redlof $\cdot$ M. Götz $\cdot$ M. Niederberger}

\section{Photovoice-Studie CORONA. Erfahrungen aus dem Leben der Hochrisikogruppe während der Pandemie}

\section{Zusammenfassung}

Hintergrund. Personen, die der Hockrisikogruppe angehören, sind einem deutlich erhöhten Risiko ausgesetzt bei einer Infektion mit COVID-19 („,coronavirus disease 2019") einen erschwerten Krankheitsverlauf zu entwickeln und daran zu sterben. Um die Verbreitung von COVID-19 in der Bevölkerung einzudämmen, werden situationsangepasste Maßnahmen durchgesetzt, die Änderungen der Alltagsgestaltung bewirken.

Ziel der Arbeit. Die Auseinandersetzung der Hochrisikogruppe mit den Auswirkungen der Coronapandemie, die partizipative Erforschung ihrer Alltagsgestaltung, Sorgen und Gesundheitsressourcen stehen im Zentrum dieser Studie.

Material und Methoden. Zur Analyse der Lebenswelt der Hochrisikogruppe wurde die
Photovoice-Methode im Online-Format mit drei Workshops eingesetzt. Sieben Hochrisikopatient:innen mit unterschiedlichen Grunderkrankungen schildern, was Corona für sie im Alltag bedeutet und wofür sie dennoch dankbar sind. Die Rekrutierung erfolgte über persönliche Kontakte des Forschungsteams. Ergebnisse. Die Co-Forschenden haben neun Geschichten formuliert, anhand derer ihre Dankbarkeit gegenüber des Gesundheitssystems und sozialen Umfelds zum Ausdruck kommt. Die Frage einer gesellschaftlichen Stigmatisierung von Personen mit Grunderkrankungen wird kritisch reflektiert. Die Geschichten zeigen, dass sie gesundheitskompetent handeln und persönliche Gesundheitsressourcen bewusst einsetzen, um negative Folgen auf ihre Gesundheit zu vermeiden und ihr Wohlbefinden zu steigern.

Diskussion. Die Co-Forschenden erweisen sich als gesundheitskompetent und in der Lage, ihr Wohlbefinden positiv zu beeinflussen. Es deutet sich jedoch an, dass der gesellschaftliche Umgang mit vulnerablen Gemeinschaften weiter zu erforschen ist, insbesondere mit Blick auf Diskriminierungsprozesse und einer bedarfsgerechten Gesundheitsversorgung.

\section{Schlüsselwörter}

COVID-19 · Chronische Grunderkrankung .

Gesundheitskompetenz · Vulnerable

Zielgruppen · Wohlbefinden

\section{Photovoice study CORONA. Life experiences of the high-risk group during the pandemic}

\section{Abstract}

Background. People who belong to the highrisk group are exposed to develop a more severe course of the coronavirus disease and have an increased risk of death if they are infected by COVID-19. To limit the spread of COVID-19 among the population, situationally appropriate regulations are enforced that change the way people live their daily lives. Objective. How does the high-risk group deal with the impact of the coronavirus pandemic, the participatory research of their everyday organization, concerns and own health resources, are the focus of this study. Material and methods. The photovoice method was used in an online format to analyze the living environment of the high-risk group. In this context three online workshops were conducted. Seven high-risk patients affected by different chronic diseases describe with pictures and stories what COVID-19 means to them and what they are grateful for in this exceptional situation. The recruitment was done by personal contact of the research team with potential participants.

Results. The co-researchers have formulated nine stories expressing their feelings of gratitude towards the healthcare system and their social environment. The question of a social stigmatization of persons with underlying diseases is critically reflected. The high-risk patients act in a healthcompetent way, consciously apply personal health resources, such as social contacts or health-promoting daily routines, to avoid negative consequences on their health and to increase their well-being.

Discussion. The co-researchers show a healthcompetent lifestyle and are able to influence their well-being in a positive way; however, there are indications that the long-term approach to vulnerable communities needs to be discussed, especially concerning discrimination processes and a needs-based health care.

Keywords COVID-19 · Chronic disease · Health literacy Vulnerable target group - Well-being kutiert und reflektiert. Die wissenschaftliche Auswertung verfolgte das Ziel, über die formulierten Geschichten der CoForschenden hinaus einen tiefgründigen Einblick zu bekommen und auch Dinge zu erfassen, die nicht direkt von den Co-Forschenden in den Geschichten benannt werden. Den Kodierleitfaden, der zur Qualitativen Inhaltsanalyse entwickelt wurde, ist in - Tab. 2 einsehbar.

Das Ergebnis der Auswertung wurde anschließend an die Co-Forschenden, mit der expliziten Bitte etwaige Änderungswünsche einzubringen, übermittelt. So kamen letztendlich neun Kategorien zustande, auf Basis derer die neun Geschichten mit dazugehörigen Fotos formuliert wurden (•Tab. 3). Drei dieser Geschichten zeigen • Infobox 1, 2 und 3.

\section{Ergebnisse zu Forschungs- frage 1 "Wofür bin ich dankbar?"}

Quer über die Geschichten zeigt sich die Dankbarkeit auf fünf Ebenen:

- Die Co-Forschenden sind über geltende Coronaschutzmaßnahmen informiert, verstehen deren Relevanz bzw. Umsetzung und handeln danach. Sie haben sich im Laufe ihrer Erkrankung die Fähigkeiten ange- 


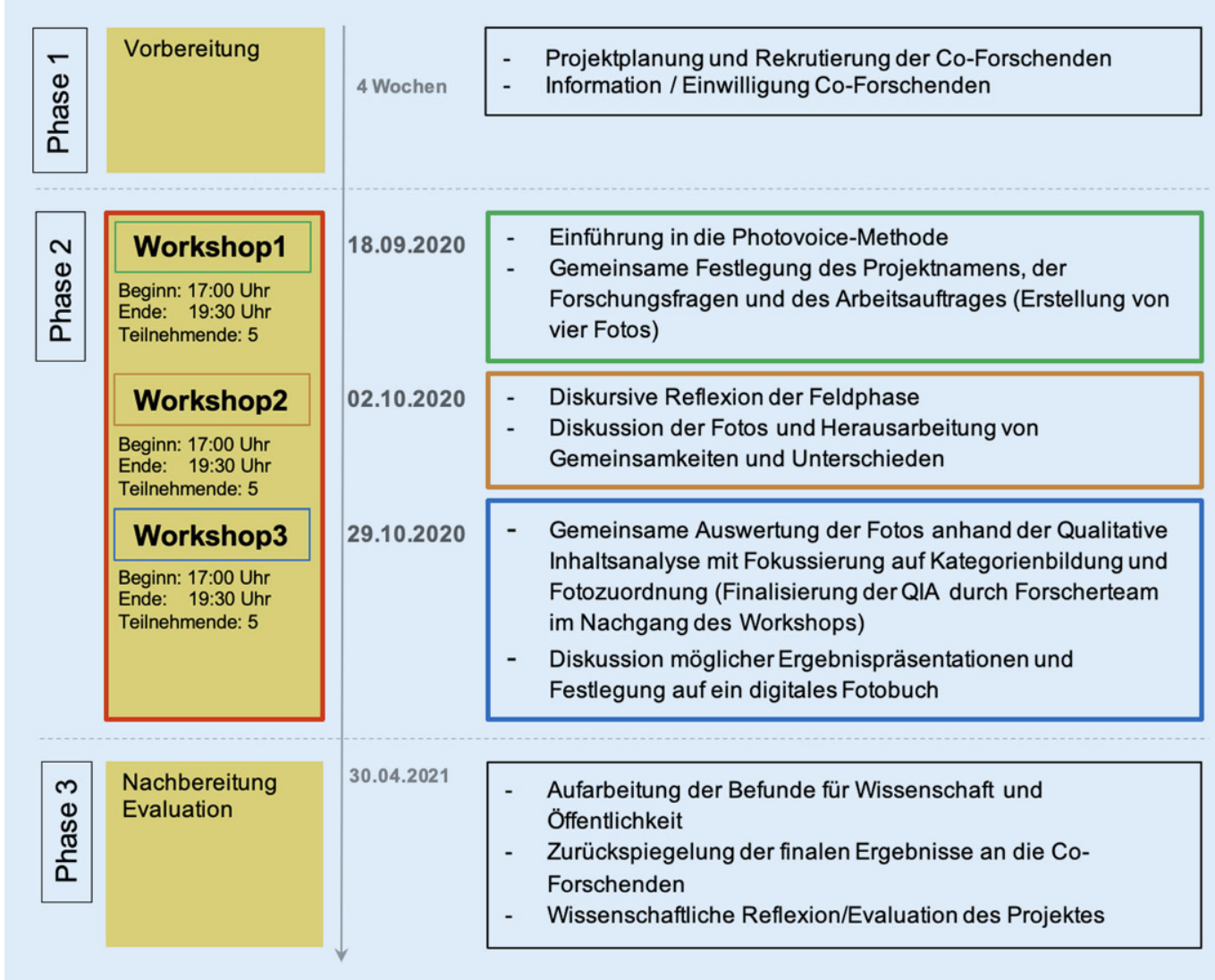

Abb. $1<$ Schematische Darstellung des Projektverlaufs der Photovoice-Studie CORONA

eignet, sich gesundheitskompetent zu verhalten und persönliche Gesundheitsressourcen zur Förderung ihres Wohlbefindens zu nutzen. Sie sind dankbar, dass sie auf außergewöhnliche Lebensbedingungen flexibel, resilient und achtsam reagieren können. Dies schaffen sie dank individueller Bewältigungsstrategien, wie einer stets reflektierten selbstfürsorgenden Alltagsgestaltung und dem Ausprobieren neuer Hobbys unter Einhaltung der Coronaregelungen.

- Die Co-Forschenden sind dankbar für ihr soziales Umfeld und sind in der Lage, eine gewisse Stabilität ihrer sozialen Kontakte während der Pandemie aufrechtzuhalten. Um mit ihren Großeltern in Kontakt zu bleiben, erläutern sie ihnen die Nutzung digitaler Tools. Dennoch räumen die Co-Forschenden ein, dass ihnen die regelmäßigen und ungezwungenen persönlichen Treffen sehr fehlen.

- Die Co-Forschenden sind dankbar für die Berücksichtigung ihrer gesundheitlichen Belange in der Coronapandemie durch die Gesellschaft. In der Zeit vor Corona machten sie Erfahrungen mit Diskriminierung bzw. Stigmatisierung. In der Ausnahmesituation haben sie nun den Eindruck, dass ihre Gesundheit für Politik und Bevölkerung ein schützenswertes Gut darstellt. Sie kritisieren und verurteilen zwar das Verhalten der „Coronaleugner:innen“, nehmen diese aber als Ausnahme wahr.

- Die Co-Forschenden sind dankbar für die Schutzmaßnahmen, die die Politik auf den Weg gebracht hat, um insbesondere Hochrisikopatient:innen vor einer COVID-19Infektion zu bewahren. Sie sind überrascht wie weitreichend diese sind und dass selbst, die bis dato unantastbare Schulpflicht, außer Kraft gesetzt wurde. Die Co-Forschenden berichten sogar von Erleichterungen in ihrem Alltag, die mit den Maßnahmen einhergehen. Durch die Möglichkeit im Homeoffice zu arbeiten, brauchen sie sich nicht vor Kolleg:innen zu rechtfertigen, wenn sie eine Pause benötigen. Bei Arztbesuchen sind die Wartezeiten kürzer und aufgrund der Abstandsregelungen haben sie keine Angst mehr, sich bei Patient:innen mit einer anderen Erkrankung anzustecken.

- Die Co-Forschenden sind auch in der Coronazeit in die medizinische Versorgungsstrukturen eingebettet, d.h. sie nehmen regelmäßige Untersuchungen bzw. Therapien wahr. Sie sind dankbar für die stabilen Versorgungsstrukturen und die Menschen, die sich im Gesundheitsbereich engagieren, unabhängig vom eigenen Risiko einer COVID-19-Erkrankung. Sie haben insgesamt den Eindruck, dass ihre Gesundheitsversorgung bedarfsgerechter erfolgt als vor der Pandemie.

Die Dankbarkeit der Co-Forschenden zeigt sich auf individueller, sozialer und struktureller Ebene. Die Co-Forschenden fühlen sich von Gesellschaft und 
Tab. 2 Kodierleitfaden der qualitativen Inhaltsanalyse

\begin{tabular}{|c|c|c|}
\hline Hauptkategorie & Unterkategorie & Erläuterung \\
\hline \multirow{5}{*}{$\begin{array}{l}\text { HK1: } \\
\text { Eigene Lebenssi- } \\
\text { tuation als Hoch- } \\
\text { risikopatient:in }\end{array}$} & - & $\begin{array}{l}\text { Persönliche Erfahrungen, Meinungen und Ver- } \\
\text { halten als Hochrisikopatient:in }\end{array}$ \\
\hline & $\begin{array}{l}\text { UK1: } \\
\text { Selbstverantwortung/ } \\
\text { persönlicher Umgang } \\
\text { mit Corona (hohe Ge- } \\
\text { sundheitskompetenz) }\end{array}$ & $\begin{array}{l}\text { Der individuelle Umgang mit den Coronamaß- } \\
\text { nahmen und Hinweise zur Gesundheitskompe- } \\
\text { tenz der Hochrisikopatient:innen }\end{array}$ \\
\hline & $\begin{array}{l}\text { UK2: } \\
\text { Eingeschränkter Kon- } \\
\text { takt zu Familie und } \\
\text { Freunden }\end{array}$ & $\begin{array}{l}\text { Hinweise zur Rolle der Freunde und Familie } \\
\text { während der Coronazeit, die sowohl positiv wie } \\
\text { auch negativ sein können }\end{array}$ \\
\hline & $\begin{array}{l}\text { UK3: } \\
\text { Soziale Isolation und } \\
\text { Einsamkeit }\end{array}$ & $\begin{array}{l}\text { Hinweise zu persönlichen Barrieren, Belastun- } \\
\text { gen und negativen Emotionen während der } \\
\text { Coronazeit }\end{array}$ \\
\hline & $\begin{array}{l}\text { UK4: } \\
\text { Notwendigkeit von } \\
\text { Achtsamkeit und Selbst- } \\
\text { fürsorge in einer Aus- } \\
\text { nahmesituation }\end{array}$ & $\begin{array}{l}\text { Hinweise zu individuellen Ressourcen während } \\
\text { der Coronazeit }\end{array}$ \\
\hline
\end{tabular}

Ankerbeispiel

HK2:

Positive Aspekte

von Corona

Durch die Hochrisikogruppe positiv bewertete Sachverhalte und Aktivitäten, die während der Coronazeit festgestellt und entdeckt wurden

Hinweise zur Veränderung der Freizeit-

UK1:

Neue Alltags- und Freizeitgestaltung unter Coronabedingungen

UK2:

Bedeutungsgewinn technischer Kommunikationswege

UK3:

Verständnis für die Schutzmaßnahmen und das Mitwirken der gesamten Bevölkerung

HK3:

gesamten Bevökerung

aktivitäten und Verhaltensweisen der Hochrisikopatienten während der Coronazeit

Hinweise zur vermehrten Nutzung von technischen Kommunikationsmitteln und deren Vorteile während der Coronazeit

Hinweise zur Wahrnehmung der Hochrisikopatienten von positiven Umständen und Wertschätzung der Gesundheit und Verständnis für die Schutzmaßnahmen innerhalb verschiedener Lebenswelten während der Coronazeit

Wahrnehmen von gesellschaftlicher Akzeptanz und Respekt durch die Hochrisikopatient:innen während der Coronazeit

„Wir können jedoch noch ein wenig Einfluss darauf nehmen, indem wir die Hygieneregeln beachten, außerdem muss man nicht unter die Leute gehen" (Margit; WS 2)

"Mittlerweile kann man den Tisch zwar decken, aber es kommt niemand und man sitzt alleine da. Vermutlich weil jeder aus Angst zuhause bleibt" (Theo; WS 2)

„ICh fühle mich isoliert" (Josef; WS 2)

„Dankbar bin ich vor allem dafür, dass ich raus gehen kann in unseren Garten" (Margit; WS 2)

„Aber jetzt sind wir so drei, vier Kumpels, die dann einfach zusammen spazieren gehen und über alles Mögliche uns austauschen - Früher saßen wir eben immer zusammen bei einem Bier" (Leo; WS 2)

"Wir haben meiner Oma ein Handy besorgt und ihr erklärt, wie es funktioniert und das Video-Chatten funktioniert jetzt schon richtig gut" (Leo; WS 2)

"Klar gibt es in der Gesellschaft Menschen, die ein besonderes Risiko haben an Corona zu erkranken und für diese wären die Auswirkungen wahrscheinlich dramatischer als für andere und deswegen brauchen sie einen besonderen Schutz" (Regina; WS 2)

"Für mich habe ich das so erfahren, dass die Gesellschaft mir Schutzraum gewährt hat, dass ich beispielsweise im Homeoffice arbeiten konnte" (Regina; WS 2)

Subjektive Bewertung und Wahrnehmung des deutschen Gesundheitssystems während des Coronazeit durch die Hochrisikopatient:innen

"ICh denke, dass nicht allen solche Sauerstoffgeräte zur Verfügung stehen und bin einmal mehr dankbar in Deutschland mit einem guten Gesundheitssystem zu leben" (Regina; WS 2)

Politik wahrgenommen, wertgeschätzt und beschützt. Sie sind in der Lage, ihren Coronaalltag achtsam und gesundheitsfördernd zu gestalten.

\section{Ergebnisse zu Forschungs- frage 2 "Was bedeutet Corona für mich?"}

Die Co-Forschenden skizzieren verschiedene Aspekte, die sich durch die Coronapandemie für sie verändert haben.
- Auf der einen Seite fühlen sich die Co-Forschenden ausgeglichener, weil sie sich mehr an der frischen Luft bewegen und weniger beruflichen Stress spüren. Gleichzeitig beschreiben sie Gefühle der Einsamkeit. Der eingeschränkte soziale Kontakt und die Ungewissheit bzw. Machtlosigkeit darüber, wie lange die Coronapandemie anhält, wird als belastend beschrieben.
- Die Co-Forschenden führen die Coronaschutzmaßnahmen auf die Berücksichtigung der Belange von Hochrisikopatient:innen zurück und empfinden dies als etwas Neues, was sie in dieser Form nicht erwartet hätten. Eine Reflexion, inwieweit dies Bestand hat und ob auch andere Gründe bei der Entwicklung von Schutzmaßnahmen eine Rolle spielen, findet nicht statt. 
Tab. 3 Übersicht zur Photovoice-Studie CORONA

\begin{tabular}{|c|c|}
\hline \multicolumn{2}{|c|}{ Die Photovoice-Studie auf einen Blick } \\
\hline Themenfeld & Gesundheit von Hochrisikopatient:innen in der Coronazeit \\
\hline \multirow[t]{2}{*}{ Forschungsfragen } & Wofür bin ich dankbar? \\
\hline & Was bedeutet Corona für mich? \\
\hline Laufzeit & $06 / 2020-03 / 2021$ \\
\hline \multirow{2}{*}{$\begin{array}{l}\text { Methodisches } \\
\text { Vorgehen }\end{array}$} & Durchführung von drei Online-Workshops \\
\hline & $\begin{array}{l}\text { Regelmäßiger telefonischer Kontakt zwischen Forschenden und Co-For- } \\
\text { schenden }\end{array}$ \\
\hline \multirow[t]{10}{*}{ Ergebnisse } & Formulierung von neun Geschichten, inklusive Fotos mit den Titeln: \\
\hline & 1. Hohe Gesundheitskompetenz \\
\hline & $\begin{array}{l}\text { 2. Notwendigkeit von Achtsamkeit und Selbstfürsorge in einer Ausnahmesi- } \\
\text { tuation }\end{array}$ \\
\hline & 3. Vorurteilsfreie Fremdwahrnehmung von Hochrisikopatient:innen \\
\hline & 4. Hohe Wertschätzung des deutschen Gesundheitssystems \\
\hline & $\begin{array}{l}\text { 5. Verständnis für die Schutzmaßnahmen und das Mitwirken der gesamten } \\
\text { Bevölkerung }\end{array}$ \\
\hline & 6. Neue Alltags- und Freizeitgestaltung unter Coronabedingungen \\
\hline & 7. Soziale Isolation und Einsamkeit \\
\hline & 8. Bedeutungsgewinn technischer Kommunikationswege \\
\hline & 9. Eingeschränkter Kontakt zu Familie und Freunden \\
\hline
\end{tabular}

- Corona bedeutet für die Co-Forschenden die Notwendigkeit eines stets reflektierten und bewussten $\mathrm{Ge}$ sundheitsverhaltens. Das Risiko einer Infektion mit COVID-19 nehmen sie als permanente Bedrohung für ihr Leben wahr, vor der sie sich aber auch selbst schützen können.

- Der Alltag der Co-Forschenden hat sich in der Coronazeit verändert. Sie probieren neue Hobbys aus, halten sich mehr in der Natur auf, nehmen sich Zeit für sich und beschreiben sich als achtsam. Sie haben sich mit den veränderten Bedingungen arrangiert und selbstbestimmt neue Alltagsroutinen etabliert. Gleichzeitig betonen sie den Wunsch wieder mehr zu unternehmen, beispielsweise andere Länder zu bereisen.

Die Co-Forschenden bleiben in ihren Darstellungen trotz des Bewusstseins des hohen persönlichen Gesundheitsrisikos positiv. Negative Gefühle formulieren sie zwar, aber immer im Verhältnis zum Risiko eines schwerwiegenden Verlaufs einer möglichen COVID-19-Erkrankung.

\section{Ergebnisse der Evaluation}

Evaluiert wurde das Projekt anhand eines schriftlichen Fragebogens mit offenen und geschlossenen Items (zu Beginn und am Ende des Projekts). Zudem erfolgte ein direktes Nachfragen innerhalb der Workshops sowie bei den bilateralen Gesprächen. Hierbei wurden eine Meinungsabfrage und ein Stimmungsbild unter Zuhilfenahme der Reflexionsmethoden erhoben und im Anschluss inhaltsanalytisch zusammengefasst. Die Auswertung der Projektevaluation durch die Co-Forschenden zeigt eine positive Einschätzung, auch im Hinblick auf den Grad der Beteiligung. Auf die offene Frage, was sie aus dem Forschungsprojekt mitnehmen, schreiben sie u. a.:

- „Ich habe gemerkt, dass es nicht nur mir allein so geht [...]. Daraus kann ich auch für mich Kraft schöpfen." Magdalena

- „Am wichtigsten fand ich die Erfahrung, wie schwierig manche aus unserem Projekt die jetzige Situation erleben und sich nicht darüber

- „Wenn ich mich an die Regeln halte, könnte man die Krise halbwegs gut überstehen." - Theo beschweren.“ - Margit

\section{Diskussion}

Die Coronapandemie erweist sich für die Co-Forschenden, wie auch für andere Bevölkerungsgruppen, als eine psychische und soziale Herausforderung $[1,18]$. Doch dank individueller Gesundheitsressourcen mildern sie negative Auswirkungen auf ihre Gesundheit ab und steigern bewusst ihr Wohlbefinden [30]. Nach der Terminologie des BelastungsBeanspruchungs-Modells [21] erlauben ihnen die individuellen Ressourcen innerhalb der geltenden Rahmenbedingungen ein Gefühl der Situationskontrolle, was ihre psychische Beanspruchung zum Zeitpunkt der Studie, also zwischen dem ersten und zweiten Lockdown, relativ gering hält. Auch frühere Studien zeigen, dass Personen mit chronischer Erkrankung eine positive Lebenseinstellung formulieren [6] und dies, obwohl ihre gesundheitsbezogene Lebensqualität gegenüber Personen ohne chronische Erkrankung als eingeschränkt gilt [23, 29]. Die Gesundheitskompetenz chronisch kranker Personen erweist sich als eine zentrale Gesundheitsressource in der Coronapandemie. Ein aktueller Review zeigt, dass zu diesem Thema bisher wenige empirische Daten vorliegen und weiterer Forschungsbedarf besteht [5].

Dennoch formulieren die Co-Forschenden auch Gefühle der permanenten Bedrohung und Machtlosigkeit. Das Risiko einer Ansteckung schwebt als „Damoklesschwert“ über ihnen und wird als bedrückend wahrgenommen [19]. Ambivalent sind die Darstellungen der Co-Forschenden beim Punkt Einsamkeit. Einerseits schaffen es die CoForschenden, den sozialen Kontakt zu einzelnen Personen aufrechtzuerhalten, andererseits fehlen ihnen persönliche Treffen. Vergleichbare Befunde zeigen auch andere Studien über die Folgen der sozialen Einschränkungen in der Coronapandemie [14].

Neben den Befunden zum aktuellen Wohlbefinden wird deutlich, dass die Hochrisikopatient:innen ein gesellschaftliches Umdenken hinsichtlich vulnerabler Gemeinschaften wahrnehmen [9]. In einer Zukunftsstudie [4] zu den Folgen der Coronapandemie betonen Expert:innen aus Wissenschaft, Wirtschaft 
Infobox 1 Kategorie 1: Hohe Gesundheitskompetenz - Mit gutem Wissen voran

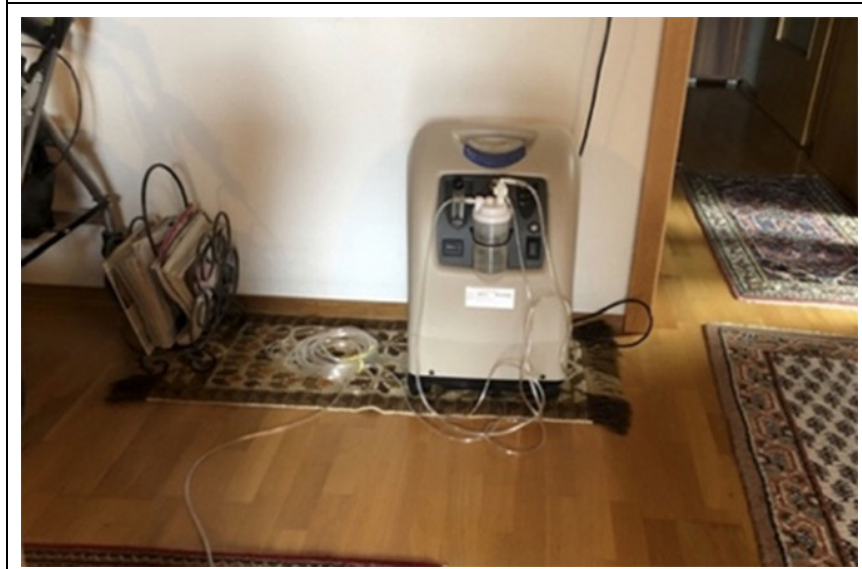

"Mein Sauerstoffgerät hilft mir dabei besser zu atmen und auch zu überleben. Aufgrund meines Lungenödems bekomme ich manchmal sehr schlecht Luft. Das Gerät erinnert mich immer wieder daran, wie gefährlich eine Infektion mit dem Coronavirus für mich wäre. Corona könnte für mich lebensbedrohlich sein. Aber ich bin mir bewusst, dass ich in dieser Situation auch selbst Verantwortung übernehmen kann, um mich vor einer Infektion zu schützen. Ich bleibe zuhause und isoliere mich selbst. Ich kenne die Hygienemaßnahmen, trage draußen eine Maske und finde auch, dass man nicht zwingend unter die Leute gehen muss. Auch Besuch empfange ich nur noch selten und wenn, dann halte ich trotzdem einen großen Abstand ein. Klar würde ich gerne öfter raus gehen, oder meine Familie und Freunde treffen wollen, aber andererseits sollte ich Abstand halten, um mich selbst und andere weniger zu gefährden. Diese Regeln und Auflagen verstehe ich. Ich kann mich selbst vor Corona schützen. Ich muss nicht darauf vertrauen, dass andere die Regeln einhalten. Dadurch habe ich weniger Angst und denke, dass ich diese schwere Zeit überstehen kann." - Josef, 83 Jahre, leidet an einer Lungenfunktionsstörung und Diabetes.

Infobox 2 Kategorie 3: Vorurteilsfreie Fremdwahrnehmung von Hochrisikopatient:innen - Verständnis füreinander

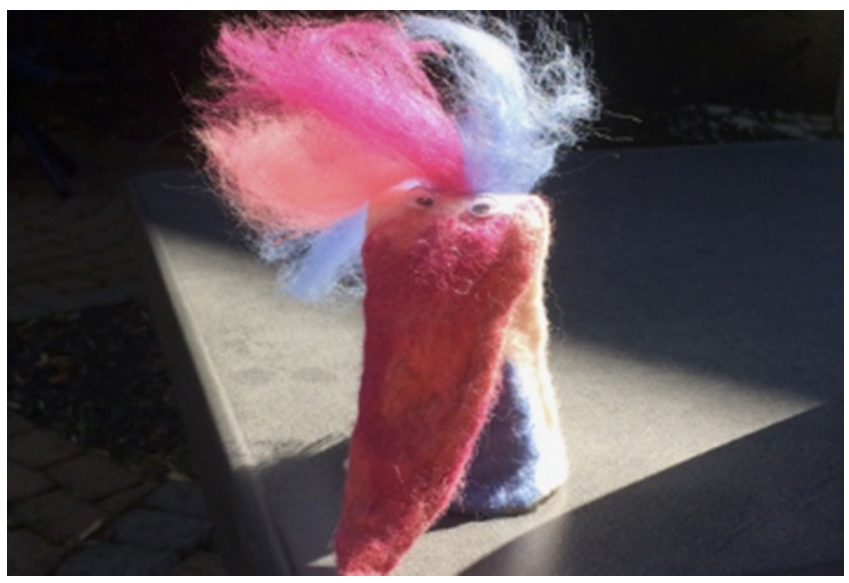

„Diesen Paradiesvogel habe ich selbst gefilzt. Er steht im Licht und leuchtet fröhlich. Corona bedeutet für mich, dass auch Paradiesvögel wie ich wichtig für die Gesellschaft sind und unsere gesundheitlichen Interessen wahrgenommen werden. Denn durch meine chronische Erkrankung gelte ich als Hochrisikopatientin und bin ungewollte ins Rampenlicht gerückt. Aber das ist gut so. Für mich als chronisch Kranke ist in dieser Zeit das Leben sogar leichter geworden. Ich muss mich nicht mehr umständlich erklären, dass ich mehr Rückzug brauche. Ich darf nun im Homeoffice arbeiten. Ich werde geschützt. Ich kann offen zu meiner Krankheit stehen, ohne dafür negativ stigmatisiert zu werden. Ich hoffe, dass dies auch nach Corona so bleibt." - Regina, 45 Jahre, leidet an einer primärsklerosierenden Cholangitis und einer Zystenniere. und Sozialverbänden die Notwendigkeit einer zukünftig verschärften Diskussion über "lebenswertes Leben“ sowie Gesundheitsleistungen für vulnerable Gruppen.

Auf einer methodischen Ebene verdeutlichen die Erfahrungen, dass Photovoice auch als Online-Format durchführbar ist [3]. Jedoch fokussiert sich der Austausch der Co-Forschenden während der Workshop-Zeit auf das Forschungsthema und damit sinkt die Chance für den Aufbau dauerhafter sozialer Kontakte. Im Hinblick auf eines der zentralen Ziele der PGF, nämlich der Stärkung vulnerabler Communities [26], kann dies kritisch gesehen werden.

\section{Limitationen}

Die Ergebnisse der Photovoice-Studien stellen eine Momentaufnahme aus dem ersten Jahr der Coronapandemie vor der Verfügbarkeit eines Impfstoffs dar. $\mathrm{Ob}$ die eher positiven Befunde über die gesamte Dauer bleiben, ist unklar. Trotz des niederschwelligen Zugangs, dem vertrauensvollen Verhältnis und der Offenheit der Co-Forschenden untereinander, kann nicht ausgeschlossen werden, dass aufgrund von sozialer Erwünschtheit, Angst oder Scham bewusst Aspekte zurückgehalten wurden. Inwieweit die Ergebnisse auf Hochrisikopatient:innen mit einer eher geringen Gesundheitskompetenz zutreffen, wäre weiter $\mathrm{zu}$ untersuchen.

\section{Fazit für die Praxis}

- Menschen mit chronischen Erkrankungen unterliegen im Alltag dem Risiko von Stigmatisierung und Diskriminierung. In der Coronapandemie haben die befragten Hochrisikopatient:innen ein höheres gesellschaftliches Interesse und ein gewisses Verständnis für ihre gesundheitlichen Belange erfahren. - Möglicherweise hat Corona die Bevölkerung für die gesundheitlichen Belange vulnerlabler Gruppen sensibilisiert. Dies kann sich zukünftig auf die Umsetzung und Akzeptanz gesundheitsförderlicher bzw. präventiver Interventionen auf Verhaltens- 


\section{Infobox 3 Kategorie 5: Verständnis für die Schutzmaßnahmen und das}

Mitwirken der gesamten Bevölkerung - Eine Hand schützt die andere

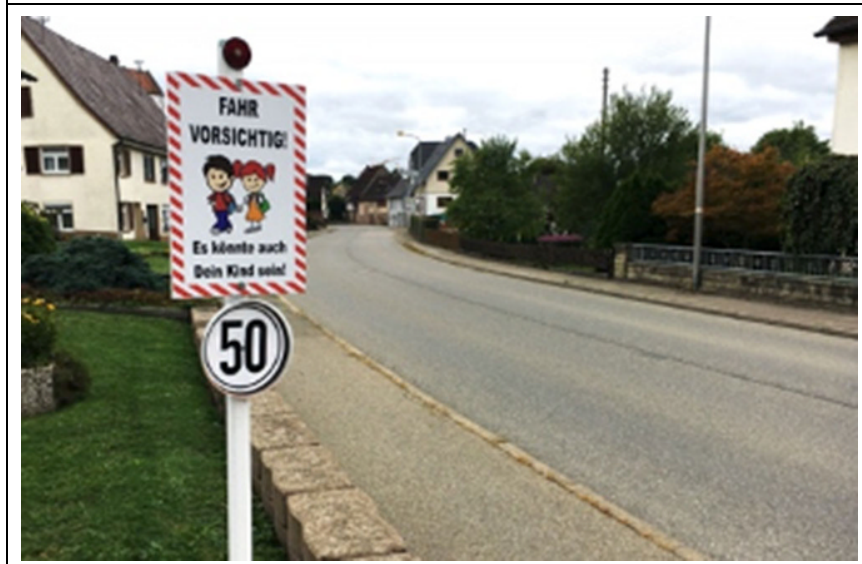

„Das Problem ist, dass jeder ein potenzieller Überträger des Coronavirus sein kann, auch wenn die Person das selbst nicht weiß. Das verunsichert mich und zeigt mir, dass wir gegen das Virus nur gemeinsam ankommen können. Ich bin positiv überrascht darüber, was alles unternommen wurde, um Corona einzudämmen und gerade auch Hochrisikopatient:innen wie mich zu schützen. Jeder kennt vermutlich wenigstens eine Person, bei der man Angst hat, dass sie schwer an Corona erkranken kann. Aber noch mehr erstaunt mich, dass es Menschen gibt, die sich aufopfern und ohne an die eigenen Folgen zu denken, sich um Hochrisikopatient:inenn wie mich kümmern. Ich denk dabei an Pflegekräfte, vor allem an meine Enkelin. Sie ist Krankenschwester und kümmert sich um die Schwachen und Kranken. Ich hoffe, dass auch weiterhin mehr auf die Gesundheit und gegenseitig Aufeinander geachtet wird." - Franziska, 79 Jahre, leidet an Asthma und einer Herzschwäche.

und Verhältnisebene als eine wichtige Grundlage erweisen.

- Partizipative Forschungsansätze erweisen sich auch in Ausnahmesituationen als geeignet, um die Co-Forschenden zur Selbstreflexion des eigenen Verhaltens anzuregen, die Gesellschaft für die Bedarfe unterschiedlicher Communities zu sensibilisieren und dadurch zum Abbau gesundheitlicher Ungleichheiten beizutragen.

\section{Korrespondenzadresse}

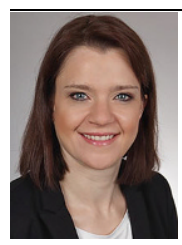

Prof. Dr.

Marlen Niederberger

Pädagogische Hochschule Schwäbisch Gmünd,

Forschungsmethoden in der Gesundheitsförderung und Prävention Oberbettringer Straße 200, 73525 Schwäbisch Gmünd, Deutschland marlen.niederberger@phgmuend.de
Forschenden Josef bedanken, welcher leider im April 2021 nach Beendigung der Studie, verstorben ist.

Funding. Open Access funding enabled and organized by Projekt DEAL.

\section{Einhaltung ethischer Richtlinien}

Interessenkonflikt. S. Redlof, M. Götz und M. Niederberger geben an, dass kein Interessenkonflikt besteht.

Für diesen Beitrag wurden von den Autoren keine Studien an Menschen oder Tieren durchgeführt. Für die aufgeführten Studien gelten die jeweils dort angegebenen ethischen Richtlinien. 


\section{Originalarbeit}

\section{Anhang}

\section{Tab. 4 Ablauf-Workshop 1}

\begin{tabular}{|c|c|c|}
\hline Zeit & Was? & Notizen \\
\hline \multirow[t]{4}{*}{$10 \mathrm{~min}$} & \multirow{4}{*}{$\begin{array}{l}\text { Check-in und Begrüßung, } \\
\text { technische Einführung in den Videokonferenz- } \\
\text { dienst Zoom, } \\
\text { Datenschutzhinweise }\end{array}$} & Kamera an? (freiwillig) \\
\hline & & Ansprache (Akronym)? Du/Sie? \\
\hline & & Einverständniserklärung ausgefüllt und abgegeben? \\
\hline & & Einführung Zoom: Chat/Hand heben/Ton/Kamera \\
\hline $5 \min$ & Vorstellung Forschungsteam & - \\
\hline \multirow[t]{3}{*}{$20 \mathrm{~min}$} & \multirow[t]{3}{*}{ Vorstellungsrunde der Co-Forschenden } & Kurze Kennenlernrunde \\
\hline & & Kamera kann freiwillig angemacht werden \\
\hline & & $\begin{array}{l}\text { Orientierungsfragen können beantwortet werden (freiwillig) } \\
\text { - Ich bin ... } \\
\text { - Aktuelle Lebenssituation } \\
\text { - Aktuelle Beschäftigung } \\
\text { - Hobbies } \\
\text { - Was bedeutet das Photovoice-Projekt für mich? }\end{array}$ \\
\hline \multirow[t]{3}{*}{$8 \min$} & \multirow{3}{*}{$\begin{array}{l}\text { Einführung in die Sicht der Gesundheitsförde- } \\
\text { rung }\end{array}$} & Definition Gesundheit \\
\hline & & Aspekte der Salutogenese erläutern \\
\hline & & Regenbogenmodell vorstellen \\
\hline \multirow[t]{2}{*}{$10 \mathrm{~min}$} & \multirow[t]{2}{*}{ Was ist Photovoice? } & Infos zur Methode und dem Forschungsprozess \\
\hline & & Kurze Darstellung Projekt: „Hauptsache Mensch“ \\
\hline \multirow[t]{2}{*}{$10 \mathrm{~min}$} & \multirow{2}{*}{$\begin{array}{l}\text { Überblick über den Ablauf des Forschungspro- } \\
\text { jekts }\end{array}$} & Einen Überblick über das Projekt bieten \\
\hline & & $\begin{array}{l}\text { Einzelne Schritte durchgehen und erläutern (WS 1, Feldphase, WS 2, Auswertung, WS 3, } \\
\text { Präsentation der Ergebnisse) }\end{array}$ \\
\hline $10 \mathrm{~min}$ & Pause mit anschließender Atemübung & Entspannende Musik wird im Hintergrund abgespielt \\
\hline \multirow[t]{3}{*}{$25 \mathrm{~min}$} & \multirow{3}{*}{$\begin{array}{l}\text { Gemeinsame Formulierung der Forschungsfra- } \\
\text { gen und des Projektnamens }\end{array}$} & Beispielformulierungen als Anstoß verwenden \\
\hline & & Was eint die Gruppe? \\
\hline & & Logo entwerfen? (Brainstorming/ldeen sammeln) \\
\hline \multirow[t]{3}{*}{$10 \mathrm{~min}$} & \multirow[t]{3}{*}{ Arbeitsauftrag vorstellen (Feldphase) } & Aufgabe erläutern (Feldphase) \\
\hline & & Leitfragen erläutern und ggf. ein Beispiel geben \\
\hline & & Auf Arbeitsblatt hinweisen, welches im Anschluss an die Co-Forschenden versendet wird \\
\hline \multirow[t]{2}{*}{$20 \mathrm{~min}$} & \multirow{2}{*}{$\begin{array}{l}\text { Erläuterung ethischer/datenschutzrechtlicher } \\
\text { und technischer Aspekte des Fotografierens, } \\
\text { kreativer Umgang mit dem Medium }\end{array}$} & Auf Fotofreigabeformular hinweisen \\
\hline & & Technische und kreative Unterweisung durch einen Co-Forschenden \\
\hline $10 \min$ & Zusammenfassung (WS 1) & $\begin{array}{l}\text { Rückblick auf den Workshop: } \\
\text { - Was haben wir gelernt? } \\
\text { - Was haben wir gemeinsam erarbeitet? }\end{array}$ \\
\hline \multirow[t]{2}{*}{$5 \mathrm{~min}$} & \multirow[t]{2}{*}{ Ausblick und Verabschiedung } & Auf den nächsten Termin ausdrücklich hinweisen \\
\hline & & Nochmal eine Möglichkeit für Fragen bieten \\
\hline
\end{tabular}


Tab. 5 Ablauf-Workshop 2

Zeit Was?

10 min Check-in und Begrüßung

3 min Vorstellung der Agenda für WS 2

$20 \mathrm{~min}$ Gemeinsame Reflexion der Feldphase

5 min Überblick über den Ablauf des Forschungsprojekts

30 min Vorstellung der Fotos (Teil 1)

10 min Pause mit anschließender Atemübung

$20 \mathrm{~min}$ Vorstellung der Fotos (Teil 2)

$20 \mathrm{~min}$ Gruppendiskussion

$10 \mathrm{~min}$ Reflexion zum Austausch

$15 \mathrm{~min}$ Gemeinsamer Austausch über die Art der Ergebnispräsentation

$8 \min \quad$ Zusammenfassung (WS 2)

$7 \mathrm{~min} \quad$ Ausblick und Verabschiedung

\section{Notizen}

Kamera an? (freiwillig)

Ansprache (Akronym)?

Steckbrief schon ausgefültt und abgegeben?

Aufzeichnung - Start (Hinweis an die Co-Forschenden)

$-$

Wie verlief das Fotografieren?

Wie erging es euch bei der Aufgabe? (einfach/schwer/überraschend)

Gab es Erkenntnisse?

Einen Überblick über den weiteren Verlauf des Projekts bieten (ab WS 2):

- Was haben wir schon geschafft?

- Was liegt noch vor uns?

Vorstellung der Fotos innerhalb der Gruppe

Die Co-Forschenden können uneingeschränkt reden

Entspannende Musik wird im Hintergrund abgespielt

Siehe oben

Gemeinsamkeiten der Gruppe finden

Was eint die Community?

Jeder kann sich an der Diskussion beteiligen - keine Vorgaben!!

Fragen:

- Wie erging es euch während eurer Fotopräsentation/der gemeinsamen Diskussion?

- Seid ihr zufrieden mit eurem Ergebnis?

- Was könnt ihr mitnehmen?

- Gibt es Wünsche oder Anregungen?

Fragen:

- Wie sollen die Ergebnisse ausgearbeitet werden? (Ideen/Anregungen/Wünsche)

Ideen sammeln

Rückblick auf den Workshop:

- Was haben wir gelernt?

- Was haben wir gemeinsam erarbeitet?

Gemeinsame Terminvereinbarung (WS 3)

Nochmal eine Möglichkeit für Fragen bieten 


\section{Originalarbeit}

\begin{tabular}{|c|c|c|}
\hline Zeit & Was? & Notizen \\
\hline \multirow[t]{3}{*}{$10 \mathrm{~min}$} & \multirow[t]{3}{*}{ Check-in und Begrüßung } & Kamera an? (freiwillig) \\
\hline & & Ansprache (Akronym)? \\
\hline & & Aufzeichnung - Start (Hinweis an die Co-Forschenden) \\
\hline $3 \min$ & Vorstellung der Agenda für WS 3 & - \\
\hline $5 \min$ & Überblick über den Ablauf des Forschungsprojekts & $\begin{array}{l}\text { Einen Überblick über den weiteren Verlauf des Projekts bieten (ab WS 2): } \\
\text { - Was haben wir schon geschafft? } \\
\text { - Was liegt noch vor uns? }\end{array}$ \\
\hline \multirow[t]{2}{*}{$15 \min$} & \multirow{2}{*}{$\begin{array}{l}\text { Vorstellung der Analyse und Auswertung der Gruppen- } \\
\text { diskussion }\end{array}$} & Alle Fotos nochmal zeigen \\
\hline & & $\begin{array}{l}\text { Leitfragen stellen: } \\
\text { - Was macht euch als Community aus? } \\
\text { - Was sind Besonderheiten? } \\
\text { - Was unterscheidet euch eventuell von "Gesunden"? }\end{array}$ \\
\hline \multirow[t]{2}{*}{$30 \mathrm{~min}$} & \multirow[t]{2}{*}{$\begin{array}{l}\text { Gruppendiskussion } \\
\text { Co-Forschende diskutieren und verifizieren die gebilde- } \\
\text { ten Kategorien (Auswertung) - Teil } 1\end{array}$} & $\begin{array}{l}\text { Leitfragen: } \\
\text { - Wie soll die jeweilige Kategorie heißen? (Titel der Geschichten) } \\
\text { - Fehlt etwas? } \\
\text { - Alles korrekt? }\end{array}$ \\
\hline & & $\begin{array}{l}\text { Co-Forschende können frei ihre Meinung äußern und sind am Entschei- } \\
\text { dungsprozess beteiligt }\end{array}$ \\
\hline $5 \mathrm{~min}$ & Pause & - \\
\hline $\begin{array}{l}30 \mathrm{~min} \\
\text { Co-Forschende }\end{array}$ & $\begin{array}{l}\text { Gruppendiskussion, } \\
\text { Co-Forschende diskutieren und verifizieren die gebilde- } \\
\text { ten Kategorien (Auswertung) - Teil } 2\end{array}$ & Siehe oben \\
\hline \multirow[t]{2}{*}{$15 \min$} & \multirow{2}{*}{$\begin{array}{l}\text { Art der Ergebnispräsentation wiederholt mit der Gruppe } \\
\text { besprechen }\end{array}$} & Ideensammlung nochmals vorstellen \\
\hline & & Abstimmung über die finale Art der Ergebnispräsentation (Co-Forschende) \\
\hline $10 \mathrm{~min}$ & Logo Abstimmung & $\begin{array}{l}\text { Verschiedene Entwürfe werden dargeboten - Abstimmung durch Co- } \\
\text { Forschenden }\end{array}$ \\
\hline \multirow[t]{2}{*}{$15 \mathrm{~min}$} & \multirow[t]{2}{*}{ Stimmungsbild und Abschlussfragen } & Offene Fragen klären \\
\hline & & Stimmungsbild einholen (Stimmungsbarometer) \\
\hline $7 \mathrm{~min}$ & Abschließende Anmerkungen und Verabschiedung & DANKE für EURE Mitarbeit! \\
\hline
\end{tabular}

Open Access. Dieser Artikel wird unter der Creative Commons Namensnennung 4.0 International Lizenz veröffentlicht, welche die Nutzung, Vervielfältigung, Bearbeitung, Verbreitung und Wiedergabe in jeglichem Medium und Format erlaubt, sofern Sie den/die ursprünglichen Autor(en) und die Quelle ordnungsgemäß nennen, einen Link zur Creative Commons Lizenz beifügen und angeben, ob Änderungen vorgenommen wurden.

Die in diesem Artikel enthaltenen Bilder und sonstiges Drittmaterial unterliegen ebenfalls der genannten Creative Commons Lizenz, sofern sich aus der Abbildungslegende nichts anderes ergibt. Sofern das betreffende Material nicht unter der genannten Creative Commons Lizenz steht und die betreffende Handlung nicht nach gesetzlichen Vorschriften erlaubt ist, ist für die oben aufgeführten Weiterverwendungen des Materials die Einwilligung des jeweiligen Rechteinhabers einzuholen.

Weitere Details zur Lizenz entnehmen Sie bitte der Lizenzinformation auf http://creativecommons.org/ licenses/by/4.0/deed.de.

\section{Literatur}

1. Brakemeier E-L, Wirkner J, Knaevelsrud C, Wurm S, Christiansen H, Lueken U, Schneider S (2020) Die COVID-19-Pandemie als Herausforderung für die psychische Gesundheit: Erkenntnisse und Implikationen für die Forschung und Praxis aus Sicht der Klinischen Psychologie und Psychotherapie. Z Klin Psychol Psychother 49(1):1-31. https://doi.org/10. 1026/1616-3443/a000574

2. Bundeszentrale für gesundheitliche Aufklärung (BZgA) (2021) Coronavirus: Risiken erkennen Sicherheit erhöhen: Wie verhalte ich mich .... https://www.infektionsschutz.de/coronavirus/ wie-verhalte-ich-mich.html. Zugegriffen: 15. Apr. 2021

3. Doyumğaç I, Tanhan A, Kiymaz MS (2020) Understanding the most important facilitators and barriers for online education during COVID-19 through online photovoice methodology. International Journal of Higher Education 10(1):166-190. http://www.sciedupress.com/journal/index.php/ ijhe/article/view/19212/11828. Zugegriffen: 15. Apr. 2021

4. Glockner $H$, Grünwald $C$, Bonin D, Irmer $M$, Astor M, Klaus C, Hornik A, Spalthoff F (2020) Langfristige Chancen und Herausforderungen in Folge der Corona-Pandemie. Eine Einschätzung von Expertinnen und Experten mittels Delphi-Methode. https://www.vorausschau.
de/files/2020_Corona_Delphi_Chancen und Herausforderungen.pdf. Zugegriffen: 15. Apr. 2021

5. Griese L (2021) Gesundheitskompetenz bei chronischer Krankheit in Deutschland: Ein Scoping Review. Präv Gesundheitsf. https://doi.org/10. 1007/s11553-021-00843-y

6. Hanum L (2015) Living with chronic disease: life satisfaction differences between elderly with one and multiple chronic disease. https:// www.researchgate.net/publication/313211838 Living_with_Chronic_Disease_Life_Satisfaction Differences_between_Elderly_with_One_and_ Multiple_Chronic_Disease. Zugegriffen: 15. Apr. 2021

7. Hartung S, Wihofszky P, Wright MT (2020) Partizipative Forschung: Ein Forschungsansatz für Gesundheit und seine Methoden. Springer VS, Wiesbaden

8. Holstiege J, Akmatov MK, Kohring C, Dammertz L, Ng F, Czihal T, von Stillfried D, Bätzing J (2021) Patienten mit stark erhöhtem Risiko für einen schweren COVID-19-Erkrankungsverlauf - Kleinräumige Planungsdaten für Impfprävention und andere bevölkerungsbezogene Interventionen bei temporär begrenzten Ressourcen mit Stand Dezember 2020. https://www.versorgungsatlas. de/fileadmin/ziva_docs/116/VA_21-01-Bericht COVID-19-Risikopat_2021-02-01.pdf. Zugegriffen: 15. Apr. 2021 
9. Hölling $H$, Schlack $R$, Dippelhofer A, Kurth BM (2008) Personale familiäre und soziale Schutzfaktoren und gesundheitsbezogene Lebensqualität chronisch kranker Kinder und Jugendlicher. Bundesgesundheitsblatt Gesundheitsforschung Gesundheitsschutz 51(6):606-620. https://doi. org/10.1007/s00103-008-0537-2

10. Huebener M, Spieß CK, Siegel NA, Wagner GG (2020) Wohlbefinden von Familien in Zeiten von Corona:Eltern mitjungen Kindern am stärksten beeinträchtigt. DIW Wochenbericht 30/31:527-537. http://www.diw.de/sixcms/detail.php?id=diw 01.c.794135.de.Zugegriffen:15. Apr. 2021

11. Jackman D, Konkin J, Yonge O, Myrick F, Cockell J (2020) Crisis and continuity: rural health care students respond to the COVID19 outbreak. Nurse Education in Practice 48:102-892. https://linkinghub.elsevier.com/ retrieve/pii/S1471595320305126.Zugegriffen: 15 . Apr. 2021

12. Kämpf $P$, Winetzhammer A (2020) Distance Learning während der Corona-Krise an Österreichs Volksschulen. Medienimpulse 58(2). https:// journals.univie.ac.at/index.php/mp/article/view/ 3795/3543.Zugegriffen: 15. Apr. 2021

13. Kompetenznetz-Angeborene Herzfehler e.V. (2020) Corona-Spezial: Wichtige Patienteninformation. Patientenbrief des Vorstandes. https://www.kompetenznetz-ahf.de/patienten/ corona-spezial/corona-virus-patientenbrief-desvorstandes/.Zugegriffen: 5. Mai 2021

14. LippkeS, KellerF, Derksen C, Kötting L, RatzT,Fleig L (2021) Einsam(er) seit der Coronapandemie: Wer ist besonders betroffen?-Psychologische Befunde aus Deutschland. Präv Gesundheitsf. https://doi. org/10.1007/s11553-021-00837-w

15. Mayring P, Fenzl T (2014) Qualitative Inhaltsanalyse. In: Baur N, Blasius J (Hrsg) Handbuch Methoden der empirischen Sozialforschung. Springer, Wiesbaden, S543-556

16. Medizinische Universität Tübingen (2021) COVID19 Pandemie - Auswirkungen auf neuroonkologische Patienten und deren Angehörige. Medizin Informatik Initiative Beobachtungsstudien. https://www.medizininformatik-initiative.de/ de/projektart/beobachtungsstudien.Zugegriffen: 5. Mai 2021

17. Meyer B, Zill A, Dilba D, Gerlach R, Schumann $S$ (2021) Employee psychological well-being during the COVID-19 pandemic in Germany: a longitudinal study of demands, resources, and exhaustion (supplemental materials). https://osf. io/2dyxp/.Zugegriffen: 15. Apr. 2021

18. Ohlbrecht $H$, Anacker J, Jellen J, Lange B, Weihrauch S (2020) Zu den Auswirkungen der CoronaPandemie auf das subjektive Wohlbefinden und die Alltagsbewältigung - Ergebnisse einer Online-Befragung. Internet-Fassung Arbeitsbericht Nr. 78. https://www.soz.ovgu.de/isoz_media/ downloads/arbeitsberichte/78_vs2-p-1534.pdf. Zugegriffen: 15. Apr. 2021

19. Renn O (1989) Risikowahrnehmung: Psychologische Determinanten bei der intuitiven Erfassung und Bewertung von technischen Risiken. In: Hosermann G (Hrsg) Risiko in der Industriegesellschaft: Analyse, Vorsorge, Akzeptanz. Erlanger Universitätsverlag, Erlangen, S 167-192

20. Robert Koch-Institut (RKI) (2020) Informationen und Hilfestellungen für Personen mit einem höheren Risiko für einen schweren COVID-19-Krankheitsverlauf. https://www.rki.de/DE/Content/ InfAZ/N/Neuartiges_Coronavirus/Risikogruppen. html.Zugegriffen: 15. Apr. 2021
21. Rohmert W (1984) Das Belastungs-Beanspruchungs-Konzept. Z Arbeitswiss 38(4):193-200

22. Rommel A, Treskova-Schwarzbach M, Scholz S, Von Der Lippe E, Robert Koch-Institut (RKI) (Hrsg) (2021) Bevölkerung mit einem erhöhten Risiko für schwere COVID-19-Verläufe in Deutschland. Auswertungen der Studie GEDA 2019/2020EHIS. Gesundheitsberichterstattung des Bundes. Journal of Health Monitoring 6(S2). https://edoc. rki.de/handle/176904/7833.2. Zugegriffen: 22. Apr. 2021

23. Shofany C (2017) Quality of life among chronic disease patients. Nursing \& Care Open Access Journal4(2).https://medcraveonline.com/NCOAJ/ quality-of-life-among-chronic-disease-patients. html.Zugegriffen: 15. Apr. 2021

24. Tanhan A, Strack RW (2020) Online photovoice to explore and advocate for Muslim biopsychosocial spiritual wellbeing and issues: Ecological systems theory and ally development. Curr Psychol 39(6):2010-2025. https://doi.org/10.1007/ s12144-020-00692-6

25. Universitätsklinikum Hamburg-Eppendorf (UKE) (2020) Corona-Krise verschlechterte psychische Gesundheit von Kindern stark. kma Online COPSY Studie. https://www.kma-online.de/aktuelles/ medizin/detail/corona-krise-verschlechtertepsychische-gesundheit-von-kindern-stark-a43619.Zugegriffen: 15. Apr. 2021

26. von Unger H (2012) Partizipative Gesundheitsforschung: Wer partizipiert woran? FQS 13(1):Art. 7. https://doi.org/10.17169/fqs-13.1.1781

27. Wachtler B, Michalski N, Nowossadeck E, Diercke M, Wahrendorf M, Santos-Hövener C, Lampert T, Hoebel J, Robert Koch-Institut (RKI) (Hrsg) (2020) Sozioökonomische Ungleichheit und COVID19 - Eine Übersicht über den internationalen Forschungsstand. Journal of Health Monitoring 5(S7). https://edoc.rki.de/handle/176904/6965. Zugegriffen: 5. Mai 2021

28. Wang C, Burris MA (1997) Photovoice: concept, methodology, and use for participatory needs assessment. Health Educ Behav 24(3):369-387

29. Wang C, Pan R, Wan X, Tan Y, Xu L, Ho CS, Ho RC (2020) Immediate psychological responses and associated factors during the initial stage of the 2019 Coronavirus disease (COVID-19) epidemic among the general population in China.IJERPH 17(5):1729. https://doi.org/10.3390/ ijerph17051729

30. Wieland R, Hammes M (2008) Gesundheitskompetenz als personale Ressource. In: Mozygemba K (Hrsg) Gesundheitswissenschaften. Nutzerorientierung - ein Fremdwort in der Gesundheitssicherung? Huber, Bern, S177-190

31. Wright M,BlockM, von UngerH(2010) Partizipative Qualitätsentwicklung in der Gesundheitsförderung und Prävention. Partizipation der Zielgruppe in der Gesundheitsförderung und Prävention. Huber, Bern

32. Wright MT (2021) Partizipative Gesundheits forschung: Ursprünge und heutiger Stand. Bundesgesundheitsblatt Gesundheitsforschung Gesundheitsschutz 64:140-145. https://doi.org/ 10.1007/s00103-020-03264-y 